\title{
One-Step Piecewise Polynomial Multiple Collocation Methods for Initial Value Problems
}

\author{
By J. P. Hennart
}

\begin{abstract}
New methods are proposed for the numerical solution of systems of firstorder differential equations. On each subinterval of a given mesh of size $h$, a polynomial of degree $l$ is constructed, its parameters being determined by a multiple collocation technique. The resulting piecewise polynomial approximation is of order $O\left(n^{l+1}\right)$ at the mesh points and between them. In addition, the $j$ th derivatives of the approximation on each subinterval provide approximations of order $O\left(n^{l+1-j}\right), j=$ $1, \ldots, l$. Some of the methods proposed are shown to be $A$-stable or even strongly $A$-stable.
\end{abstract}

1. Introduction. Recently, "semidiscrete" Galerkin techniques have received considerable attention for the approximate solution of evolution equations. See, for example, [25], [10], [24], [12]. When the space variables have been integrated out, one typically has to solve a system of nonlinear differential equations. Since the space basis functions are usually taken of the finite element type, i.e. they are piecewise polynomials with local support, a more comprehensive treatment would be achieved by approximating the time behavior also by piecewise polynomials, in order to get a fully piecewise polynomial approximation.

In this paper, we develop new one-step methods for systems of nonlinear firstorder ordinary differential equations. Our basic idea is to find local $l$ th degree polynomial approximations on each subinterval of a given mesh, the free parameters being determined by a multiple collocation technique based on the two-point Taylor interpolation formula. The resulting approximations are piecewise-continuous and, when combined with "semidiscrete" Galerkin methods, they provide fully piecewise-continuous approximations to initial-boundary value problems. Earlier uses of piecewise polynomials for systems of ODE's may be found in [21], [22], [29], [16], [7], [17] , [18], [6], [1], [2]. Finite elements in space and time have been proposed in [23], [3] , $[30],[31],[5],[20],[27]$.

In Section 2, we introduce our multiple collocation techniques. Section 3 is devoted to the derivation of order of convergence results. Section 4 examines the stability properties of the proposed schemes while Section 5 exhibits some numerical results. Preliminary results have been reported in [13] and [14]. In our presentation, we shall follow [17], [18] because of the similarity in the methods. The novelty in our schemes is that, instead of collocating at distinct abscissas in each subinterval as in

Received July 15, 1975 ; revised April 12, 1976.

AMS (MOS) subject classifications (1970). Primary 34A50, 65 L05.

Key words and phrases. Initial value problems, ordinary differential equations, piecewise polynomials, collocation methods, $\boldsymbol{A}$-stability. 
[17] , [18], we allow the collocation points to coalesce at both ends of the subinterval, providing multiple collocation at the meshpoints. After this work was completed, a manuscript by J. Descloux and N. Nassif, in which similar methods are developed independently, has been brought to our attention ( $\mathrm{N}$. Nassif, personal communication, 1975).

2. Piecewise Polynomial Multiple Collocation Methods. Let us consider the solution of the following nonlinear first-order differential equation

$$
Y^{\prime}(t)=f(Y(t), t), \quad t \in\left[t_{0}, t_{N}\right],
$$

subject to the initial condition

$$
Y(0)=Y_{0} \text {. }
$$

Although a single equation is considered, the methods and theorems which will be presented carry over to systems of first-order equations. We assume that $f(Y, t)$ is of class $C^{k}, k \geqslant 0$, over $R \times\left[t_{0}, t_{N}\right]$ so that the exact solution $Y(t) \in C^{k+1}\left[t_{0}, t_{N}\right]$. Moreover $f$ and some of its derivatives (as we shall see later) are supposed to satisfy a Lipschitz condition over the same domain.

For the sake of simplicity, let $\Pi: t_{i}=i h, i=0, \ldots, N$, be a uniform mesh of meshsize $h$, although a variable mesh $\Pi$ could have been considered without affecting our arguments. Then, over each subinterval of $\Pi$, we may approximate $Y(t)$ by a polynomial $Y_{p, q}(t)$ of degree $l(l \geqslant 0)$

$$
Y(t) \simeq Y_{p, q}(t), \quad t \in\left[t_{i}, t_{i+1}\right], i=0, \ldots, N-1,
$$

uniquely and completely determined by $(l+1)$ parameters which are its value and its successive derivatives up to order $(p-1)$ at $t=t_{i}$ (if $\left.p>0\right)$ and up to order $(q-1)$ at $t=t_{i+1}$ (if $q>0$ ), with $p+q=l+1, p, q \geqslant 0$. Actually, we have

$$
Y_{p, q}(t)=\left(t-t_{i}\right)^{p} \sum_{r=0}^{q-1} \frac{B_{r}\left(t-t_{i+1}\right)^{r}}{r !}+\left(t-t_{i+1}\right)^{q} \sum_{s=0}^{p-1} \frac{A_{s}\left(t-t_{i}\right)^{s}}{s !}
$$

with

$$
A_{s}=\frac{d^{s}}{d t^{s}}\left[\frac{Y_{p, q}(t)}{\left(t-t_{i+1}\right)^{q}}\right]_{t=t_{i}}
$$

and

$$
B_{r}=\frac{d^{r}}{d t^{r}}\left[\frac{Y_{p, q}(t)}{\left(t-t_{i}\right)^{p}}\right]_{t=t_{i+1}}
$$

Equation (4) is a trivial extension of the two-point Taylor formula [8, p. 37]. The parameters $A_{s}$ and $B_{r}$ given by Eqs. (5) and (6) are linear combinations of the successive derivatives of $Y_{p, q}(t)$ at $t=t_{i}$ and $t_{i+1}$. These derivatives may be eliminated at 
both ends of the interval by multiple collocation using the original Eq. (1), namely

$$
Y_{p, q}^{(s)}\left(t_{i}\right)=f^{(s-1)}\left(Y_{p, q}\left(t_{i}\right), t_{i}\right), \quad s=1, \ldots, p-1 \quad(\text { with } p \geqslant 2),
$$

and

$$
Y_{p, q}^{(r)}\left(t_{i+1}\right)=f^{(r-1)}\left(Y_{p, q}\left(t_{i+1}\right), t_{i+1}\right), \quad r=1, \ldots, q-1 \quad(\text { with } q \geqslant 2) .
$$

This is only possible if $f$ is smooth enough, i.e. if $k \geqslant \max (p, q)-2$. This condition might seem somewhat restrictive: actually, it is sufficient that it be satisfied in a piecewise sense and in particular over each subinterval of $\pi$, which is much less restrictive. As a consequence of the collocation conditions (7) and (8), there remain at most two parameters in the expression (4) of $Y_{p, q}(t)$, namely $Y_{i+1} \equiv Y_{p, q}\left(t_{i+1}\right)$ and (eventually) $Y_{p, q}\left(t_{i}\right)$ : indeed, for reasons that will be explained later, we shall usually restrict ourselves to the cases $q=p, q=p+1$ or $q=p+2$ so that $Y_{p, q}\left(t_{i}\right)$ will appear explicitly in the expression of $Y_{p, q}(t)$ except for $l=0, p=0, q=1$ and $l=1, p=0$, $q=2$. If $Y_{p, q}\left(t_{i}\right)$ appears explicitly, its value $Y_{i}$ is known either from the initial condition $(i=0)$ or from integration over the previous interval $\left[t_{i-1}, t_{i}\right], i=1, \ldots$, $N-1$, while $Y_{i+1}$ is obtained by integration of Eq. (1) over $\left[t_{i}, t_{i+1}\right]$ :

$$
Y_{i+1}=Y_{i}+\int_{t_{i}}^{t_{i+1}} d t f\left(Y_{p, q}(t), t\right), \quad i=0, \ldots, N-1,
$$

where $Y_{p, q}(t)=Y_{p, q}\left(Y_{i}, Y_{i+1}, t\right)$.

To obtain a computational form of (9), it is necessary to perform a numerical quadrature, unless the integral may be evaluated analytically. In general, we shall use an $n$-point quadrature formula of the form:

$$
\begin{gathered}
\int_{t_{i}}^{t_{i+1}} f\left(Y_{p, q}(t), t\right) d t \cong h \sum_{j=1}^{n} w_{j} f\left(Y_{p, q}\left(\tau_{i j}\right), \tau_{i j}\right)+O\left(h^{m+2}\right), \\
\tau_{i j}=t_{i}+\theta_{j} h, \quad j=1, \ldots, n,
\end{gathered}
$$

where $w_{j}$ and $\theta_{j}$ are weights and abscissae for $[0,1]$. No matter what quadrature formula we choose, it should be accurate enough, i.e. in general $k \geqslant m \geqslant l$. As a result, (9) becomes a nonlinear equation in the only unknown $Y_{i+1}$ :

$$
Y_{i+1}=Y_{i}+h \sum_{j=1}^{n} w_{j} f\left(Y_{p, q}\left(\tau_{i j}\right), \tau_{i j}\right) \text {, }
$$

since $Y_{p, q}\left(\tau_{i j}\right)$ is a function of $Y_{i}$ and $Y_{i+1}$ only. (12) defines thus a class of discrete one-step integration methods depending on which quadrature formula is used.

The existence of a unique solution to (12) is guaranteed for $h$ sufficiently small by the following argument. First of all,

$$
\begin{aligned}
\left|Y_{i+1}-Y_{i+1}^{*}\right| & =h\left|\sum_{j=1}^{n} w_{j}\left(f\left(Y_{p, q}\left(\tau_{i j}\right), \tau_{i j}\right)-f\left(Y_{p, q}^{*}\left(\tau_{i j}\right), \tau_{i j}\right)\right)\right| \\
& \leqslant h L \sum_{j=1}^{n}\left|w_{j}\right|\left|Y_{p, q}\left(\tau_{i j}\right)-Y_{p, q}^{*}\left(\tau_{i j}\right)\right|,
\end{aligned}
$$

where $L$ is the Lipschitz constant for $f$ over $R \times\left[t_{0}, t_{N}\right]$. 
Let us rewrite (4) as

$$
Y_{p, q}(t)=\sum_{r=0}^{q-1} P_{p, q}^{r}(t) Y_{p, q}^{(r)}\left(t_{i+1}\right)+\sum_{s=0}^{p-1} Q_{p, q}^{s}(t) Y_{p, q}^{(s)}\left(t_{i}\right)
$$

where $P_{p, q}^{r}(t)$ and $Q_{p, q}^{s}(t) \in P_{l}$. Similarly,

$$
Y_{p, q}^{*}(t)=\sum_{r=0}^{q-1} P_{p, q}^{r}(t) Y_{p, q}^{*(r)}\left(t_{i+1}\right)+\sum_{s=0}^{p-1} Q_{p, q}^{s}(t) Y_{p, q}^{(s)}\left(t_{i}\right)
$$

so that

$$
Y_{p, q}(t)-Y_{p, q}^{*}(t)=\sum_{r=0}^{q-1} P_{p, q}^{r}(t)\left(Y_{p, q}^{(r)}\left(t_{i+1}\right)-Y_{p, q}^{*(r)}\left(t_{i+1}\right)\right)
$$

and

$$
\begin{aligned}
\left|Y_{p, q}\left(\tau_{i j}\right)-Y_{p, q}^{*}\left(\tau_{i j}\right)\right| & \leqslant \sum_{r=0}^{q-1}\left|P_{p, q}^{r}\left(\tau_{i j}\right)\right|\left|Y_{p, q}^{(r)}\left(t_{i+1}\right)-Y_{p, q}^{*(r)}\left(t_{i+1}\right)\right| \\
& \leqslant\left(P_{0}+\sum_{r=1}^{q-1} P_{r} L_{r}\right)\left|Y_{i+1}-Y_{i+1}^{*}\right|
\end{aligned}
$$

with

$$
P_{r}=\max _{t \in\left[t_{i}, t_{i+1}\right]}\left|P_{p, q}^{r}(t)\right|, \quad r=0, \ldots, q-1,
$$

while $L_{r}$ is the Lipschitz constant (supposed to exist) for $f^{(r-1)}$ over $R \times\left[t_{0}, t_{N}\right]$ with in particular $L_{1} \equiv L$. Using (15), (13) becomes

$$
\left|Y_{i+1}-Y_{i+1}^{*}\right| \leqslant h L\left(\sum_{j=1}^{n}\left|w_{j}\right|\right)\left(P_{0}+\sum_{r=1}^{q-1} P_{r} L_{r}\right)\left|Y_{i+1}-Y_{i+1}^{*}\right|,
$$

so that the right side of (12) appears to be a contraction mapping on $R$ when

$$
h<h_{0}\left\{L\left(\sum_{j=1}^{n}\left|w_{j}\right|\right)\left(P_{0}+\sum_{r=1}^{q-1} P_{r} L_{r}\right)\right\}^{-1} .
$$

A successive substitution iteration would consequently converge to the unique solution of (12). However, we should mention that for practical purposes a NewtonRaphson type technique would be much more efficient in presence of large Lipschitz constants, i.e. for stiff equations.

3. Orders of Convergence. In this section, discrete and continuous error bounds are derived for the methods developed hereinabove. Discrete error bounds are obtained from Henrici's theory of discrete one-step methods [15, Chapter 2]. Continuous error bounds are then obtained from the discrete ones.

First of all, in order for Henrici's theory to apply, we must show that the increment function $h \times \sum_{j=1}^{n} w_{j} f\left(Y_{p, q}\left(\tau_{i j}\right), \tau_{i j}\right)$ is Lipschitz continuous with respect to $Y_{i}$ over $R \times\left[t_{0}, t_{N}\right]$. This can be easily done by repeating the argument given at the end of Section 2 with $Y_{i}$ instead of $Y_{i+1}$. 
Then, we may prove

THEOREM 1. Assume that $f(Y, t)$ is of class $C^{k}, k \geqslant l$, over $R \times\left[t_{0}, t_{N}\right]$, and let the multiple collocation method be defined as in Section 2 for some polynomials $Y_{p, q}(t)$ of degree $l$. Then, there exists a constant $C$ such that

$$
\left|Y\left(t_{i}\right)-Y_{i}\right| \leqslant C h^{l+1}, \quad i=0, \ldots, N .
$$

Proof. We shall follow the same general method of proof as for Theorems 1 of [17], [18], except that we define the local truncation error differently, thereby correcting a technical error that exists in Hulme's proofs of the order of convergence of his methods, although his conclusions are correct. Indeed, the local truncation error is defined by

$$
\tau_{i}=\int_{t_{i}}^{t_{i+1}} f(Y(t), t) d t-h \sum_{j=1}^{n} w_{j} f\left(Y_{p, q}\left(\tau_{i j}\right), \tau_{i j}\right)
$$

where in the last term $Y_{i} \doteq Y\left(t_{i}\right)$. Thus,

$$
\begin{aligned}
\tau_{i}= & \int_{t_{i}}^{t_{i+1}} f(Y(t), t) d t-\int_{t_{i}}^{t_{i+1}} f\left(Y_{p, q}(t), t\right) d t \\
& +\int_{t_{i}}^{t_{i+1}} f\left(Y_{p, q}(t), t\right) d t-h \sum_{j=1}^{n} w_{j} f\left(Y_{p, q}\left(\tau_{i j}\right), \tau_{i j}\right),
\end{aligned}
$$

and

$$
\left|\tau_{i}\right| \leqslant\left|\int_{t_{i}}^{t_{i+1}}\left(f(Y(t), t)-f\left(Y_{p, q}(t), t\right)\right) d t\right|+O\left(h^{m+2}\right),
$$

in virtue of (10). Moreover,

$$
\begin{aligned}
& \left|\int_{t_{i}}^{t_{i+1}}\left(f(Y(t), t)-f\left(Y_{p, q}(t), t\right)\right) d t\right| \\
& \quad \leqslant \int_{t_{i}}^{t_{i+1}}\left|f(Y(t), t)-f\left(Y_{p, q}(t), t\right)\right| d t \leqslant L \int_{t_{i}}^{t_{i+1}}\left|Y(t)-Y_{p, q}(t)\right| d t
\end{aligned}
$$

To analyze this term, let us make use of the fact that whenever $f$ is independent of $Y$ and $f \in P_{l-1}$, the exact solution $Y \in P_{l}$ and $Y(t) \equiv Y_{p, q}(t)$ over any interval. This follows because the quadrature (10) is exact for polynomials of degree $m \geqslant l$ so that $Y\left(t_{i+1}\right)=Y_{i+1}$ if $Y\left(t_{i}\right)=Y_{i}$. Over each interval $Y(t)$ and $Y_{p, q}(t)$ are thus polynomials of degree $l$ satisfying the same interpolation conditions (7) and (8). By uniqueness, they are consequently equivalent, so that $L(Y) \equiv Y(t)-Y_{p, q}(t)$ is a linear functional of $Y$, equal to zero for all polynomials of degree $l$. It is a straightforward application of the Peano kernel theorem [8] that

$$
L(Y)=\frac{1}{l !} L_{t} \int_{t_{i}}^{t_{i+1}} Y^{(l+1)}(x)(t-x)_{+}^{l} d x,
$$

where

$$
(t-x)_{+}^{l} \equiv \begin{cases}(t-x)^{l}, & t \geqslant x \\ 0, & t<x\end{cases}
$$


and $L_{t}$ means the linear functional $L$ applied to the expression

$$
\int_{t_{i}}^{t_{i+1}} Y^{(l+1)}(x)(t-x)_{+}^{l} d x
$$

considered as a function of $t$. As a consequence,

$$
\left\|Y(t)-Y_{p, q}(t)\right\|_{L^{\infty}\left[t_{i}, t_{i+1}\right]} \leqslant C_{i} h^{l+1},
$$

where $C_{i}$ is some constant depending on $\left\|Y^{(l+1)}\right\|_{L^{\infty}\left[t_{i}, t_{i+1}\right]}$, and

$$
\int_{t_{i}}^{t_{i+1}}\left|Y(t)-Y_{p, q}(t)\right| d t
$$

is thus of $O\left(h^{l+2}\right)$. From (18) and (19), $\left|\tau_{i}\right| \leqslant C h^{l+2}$, where we used the fact that $m \geqslant l$. The bound (17) follows immediately from Henrici's Theorem 2.2 [15]. Q.E.D.

Continuous error bounds are given by

THEOREM 2. With the hypotheses of Theorem 1, there exist constants $C_{j}, j=$ $0, \ldots, l$, such that

$$
\left\|Y^{(j)}(t)-Y_{p, q}^{(j)}(t)\right\|_{L^{\infty}\left[t_{i}, t_{i+1}\right]} \leqslant C_{j} h^{l+1-j} .
$$

Proof. Using the same two-point Taylor interpolation formula (4) for $Y(t)$ as for $Y_{p, q}(t)$, we have

(21) $Y(t)=\sum_{r=0}^{q-1} P_{p, q}^{r}(t) Y^{(r)}\left(t_{i+1}\right)+\sum_{s=0}^{p-1} Q_{p, q}^{s}(t) Y^{(s)}\left(t_{i}\right)+R_{l}(t), \quad t \in\left[t_{i}, t_{i+1}\right]$, where $R_{l}(t)=O\left(h^{l+1}\right)$. Subtracting (14) from (21), we get

$$
\begin{aligned}
\left|Y(t)-Y_{p, q}(t)\right| \leqslant & \sum_{r=0}^{q-1}\left|P_{p, q}^{r}(t)\right|\left|Y^{(r)}\left(t_{i+1}\right)-Y_{p, q}^{(r)}\left(t_{i+1}\right)\right| \\
& +\sum_{s=0}^{p-1}\left|Q_{p, q}^{s}(t)\right|\left|Y^{(s)}\left(t_{i}\right)-Y_{p, q}^{(s)}\left(t_{i}\right)\right|+O\left(h^{l+1}\right) \\
\leqslant & \left(P_{0}+\sum_{r=1}^{q-1} P_{r} L_{r}\right)\left|Y\left(t_{i+1}\right)-Y_{i+1}\right| \\
& +\left(Q_{0}+\sum_{s=0}^{p-1} Q_{r} L_{r}\right)\left|Y\left(t_{i}\right)-Y_{i}\right|+O\left(h^{l+1}\right),
\end{aligned}
$$

where $L_{r}$ is again the Lipschitz constant for $f^{(r-1)}$ over $R \times\left[t_{0}, t_{N}\right], P_{r}$ is given by (16) and $Q_{s}$ is defined similarly by

$$
Q_{s}=\max _{t \in\left[t_{i}, t_{i+1}\right]}\left|Q_{p, q}^{s}(t)\right|, \quad s=0, \ldots, p-1 .
$$

The bound (20) for $j=0$ is then a direct consequence from (19) and (22). For $j \neq$ 0 , let us differentiate (14) and (21) $j$ times before subtracting; using $R_{l}^{(j)}(t)=$ $O\left(h^{l+1-j}\right)$, we get 


$$
\begin{aligned}
\left|Y^{(j)}(t)-Y_{p, q}^{(j)}(t)\right| \leqslant & \sum_{r=0}^{q-1}\left|P_{p, q}^{r(j)}(t)\right|\left|Y^{(r)}\left(t_{i+1}\right)-Y_{p, q}^{(r)}\left(t_{i+1}\right)\right| \\
& +\sum_{s=0}^{q-1}\left|Q_{p, q}^{r(j)}(t)\right|\left|Y^{(s)}\left(t_{i}\right)-Y_{p, q}^{(s)}\left(t_{i}\right)\right| \\
& +O\left(h^{l+1-j}\right), \quad j=1, \ldots, l .
\end{aligned}
$$

A similar argument to the one given hereinabove leads directly to the bounds (20) for $j \neq 0$. Q.E.D.

Except for the approximations $p=0, q=1$ and $p=0, q=2$ for which $Y_{p, q}\left(t_{i}\right)$ does not appear explicity in (4) and is therefore not necessarily equal to $Y_{i}$, the approximation $Y_{p, q}(t)$ of $Y(t)$ is continuous over $\left[t_{0}, t_{N}\right]$ and we have:

THEOREM 3. With the hypotheses of Theorem 1 and if $p \geqslant 1$ there exists a constant $C$ such that

$$
\left\|Y(t)-Y_{p, q}(t)\right\|_{L^{\infty}\left[t_{0}, t_{N}\right]} \leqslant C h^{l+1} .
$$

Proof. The bound (23) follows directly from Theorem 2 and the fact that $Y_{p, q}(t)$ is $C^{0}\left[t_{0}, t_{N}\right]$. Q.E.D.

4. Stability Properties. When applied to the test equation $Y^{\prime}=\lambda Y$, the methods of Section 2 give

$$
Y_{i+1}=R_{p q}(h \lambda) Y_{i}
$$

with $R_{p q}(h \lambda)=P_{p}(h \lambda) / Q_{q}(h \lambda), P_{p}(\mu)$ and $Q_{q}(\mu)$ being polynomials in $\mu$ of degree $p$ and $q$ respectively, with constant coefficients. This follows directly from the observation that the collocation conditions (7) and (8) become

$$
Y_{p, q}^{(s)}\left(t_{i}\right)=\lambda^{s} Y_{p, q}\left(t_{i}\right), \quad s=1, \ldots, p-1(\text { with } p \geqslant 2)
$$

and

$$
\left.Y_{p, q}^{(r)}\left(t_{i+1}\right)=\lambda^{r} Y_{p, q}\left(t_{i+1}\right), \quad r=s, \ldots, q-1 \text { (with } q \geqslant 2\right) .
$$

Since the method is of order $l+1$,

$$
R_{p q}(h \lambda)=e^{h \lambda}+O\left(h^{l+2}\right) .
$$

Hence $R_{p q}(\mu)$ is the $(q, p)$ entry of the table of Padé approximants to $e^{\mu}$, namely [19]:

$$
R_{p q}(\mu)=\left(\sum_{k=0}^{p} a_{k} \mu^{k}\right) /\left(\sum_{l=0}^{q} b_{l} \mu^{l}\right)
$$

with

and

$$
a_{k}=\frac{(p+q-k) ! p !}{(p+q) ! k !(p-k) !}
$$




$$
b_{l}=(-1)^{l} \frac{(p+q-l) ! q !}{(p+q) ! l !(q-l) !} .
$$

Following Dahlquist [9], a method is called $A$-stable if all its solutions tend to zero, as $i \rightarrow \infty$, when it is applied with fixed positive $h$ to the test equation $Y^{\prime}=\lambda Y$, where $\lambda$ is any complex constant with negative real part. For the one-step methods (24), this implies

$$
\left|R_{p q}(\mu)\right| \leqslant 1, \quad \forall \mu \text { complex with } \operatorname{Re}(\mu) \leqslant 0 .
$$

If, moreover, $\left|R_{p q}(\mu)\right| \longrightarrow 0$ as $\operatorname{Re}(\mu) \longrightarrow-\infty$, the one-step method considered is strongly A-stable; such a method should be particularly effective for stiff systems of equations since rapidly decaying components of the solution will be represented by rapidly decaying components in the approximate solution for any $h$. If $(25)$ is valid only for $|\arg (-\lambda)| \leqslant \alpha, \alpha \in[0, \pi / 2]$, then the corresponding method is called $A(\alpha)$ stable [28], $A(\pi / 2)$-stability being equivalent to $A$-stability. For many space discretized parabolic problems, the eigenvalues of the Jacobian matrix are real negative: in this case, $A(0)$-stability is quite sufficient. In [26], Varga has shown that all the Padé approximants to $\exp (\mu)$ with $q \geqslant p$ give rise to $A(0)$-stable one-step schemes. That the diagonal $(p=q)$ Padé approximants to $\exp (\mu)$ provide $A$-stable schemes was proved by Birkhoff and Varga [4]. Later on, Ehle [11] has shown that the Padé approximants to $\exp (\mu)$ with $q=p+1$ and $q=p+2$ lead to strongly $A$-stable schemes. Ehle has exhibited moreover some Padé approximants with $q=p+3$ which do not satisfy condition (25) and do not lead therefore to $A$-stable schemes.

All the schemes developed hereinabove are thus $A$-stable for $q=p, p+1$ or $p+2$. If $q=p+1$ or $p+2$, they are moreover strongly $A$-stable and should therefore be preferred in the case of stiff systems of differential equations. This was shown in a previous work [13] for the nuclear reactor point and space kinetics equations.

5. Numerical Experiments. In this section, we give numerical results for some sample problems. First of all, we considered the case of a single equation:

$$
Y^{\prime}(t)=Y-2 t / Y, \quad Y(0)=1, \quad Y(t)=(2 t+1)^{1 / 2}, \quad t \in[0,1],
$$

computed with some methods of Section 1, namely the simplest ones for which no derivatives of $f$ are to be evaluated, i.e. $p, q \leqslant 2$, using moreover a three-point GaussLegendre quadrature formula in (12). Table 1 exhibits the discrete error norms

$$
\|e(t ; h)\|^{\prime}=\max _{0 \leqslant i \leqslant N}\left|e\left(t_{i} ; h\right)\right|
$$

for $h=1 / 2^{N}, N=1, \ldots, 6$, with $e\left(t_{i} ; h\right)=Y\left(t_{i}\right)-Y_{i}$, as well as the computed orders of convergence (in parentheses)

$$
\alpha=\frac{\log \left[\left\|e\left(t ; h_{1}\right)\right\|^{\prime} /\left\|e\left(t ; h_{2}\right)\right\|^{\prime}\right]}{\log \left(h_{1} / h_{2}\right)} \simeq l+1
$$

based on successive meshsizes $h_{1}$ and $h_{2}$. The nonlinear equations (12) were solved by successive substitution at each step $\left[t_{i}, t_{i+1}\right]$ until $Y_{i+1}$ satisfied a relative error tolerance $10^{-11}$. 


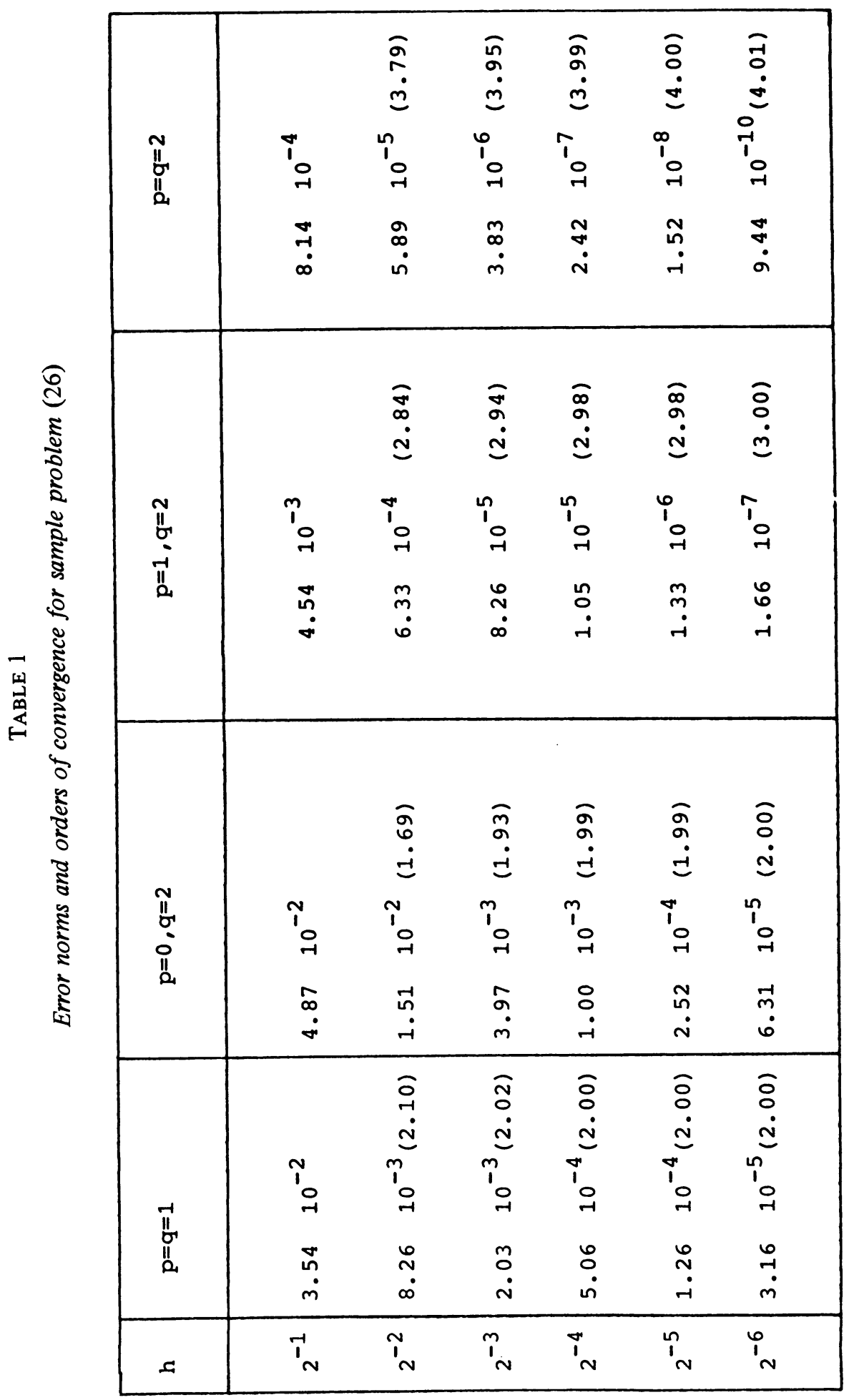


COLLOCATION METHODS

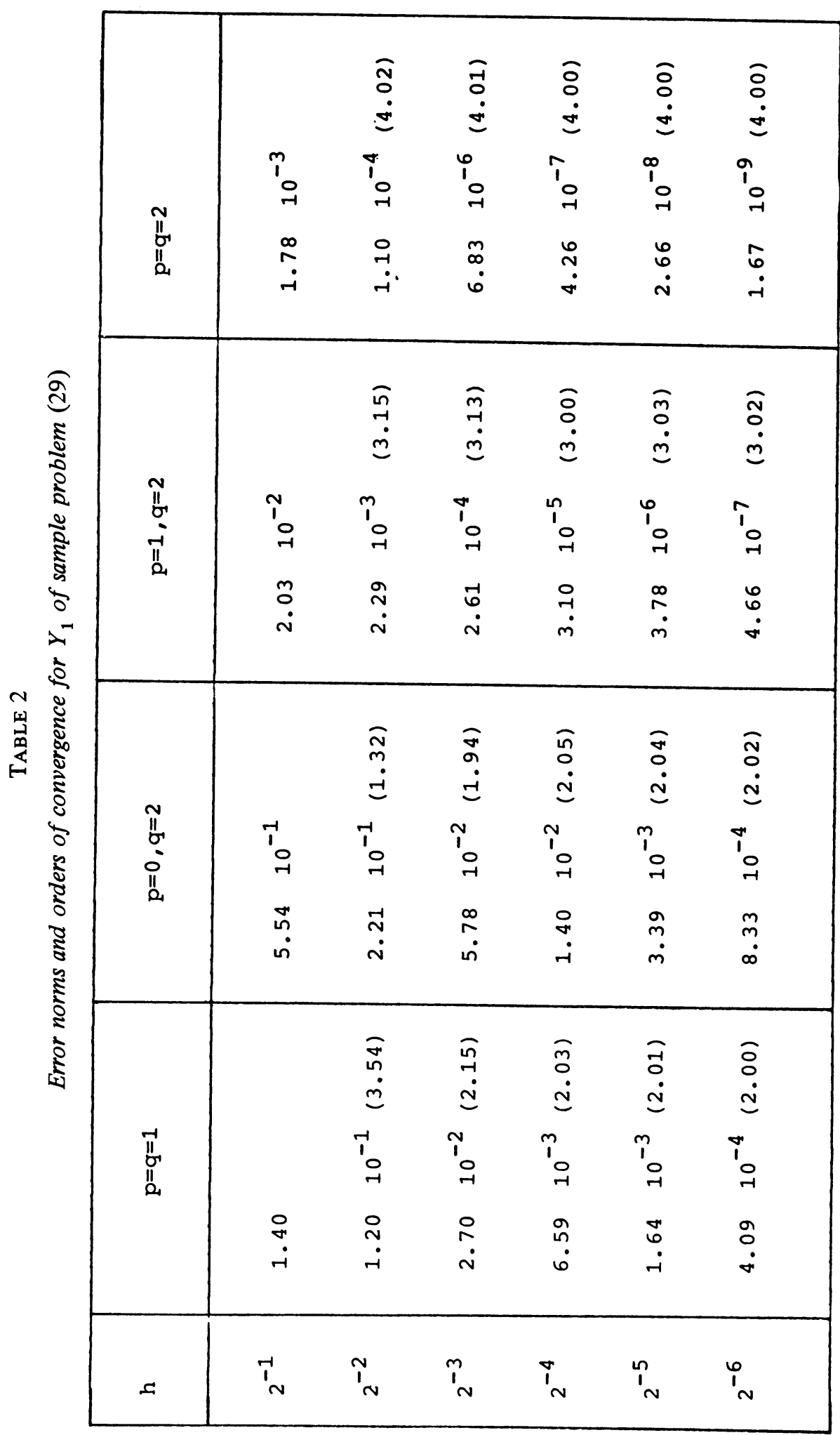




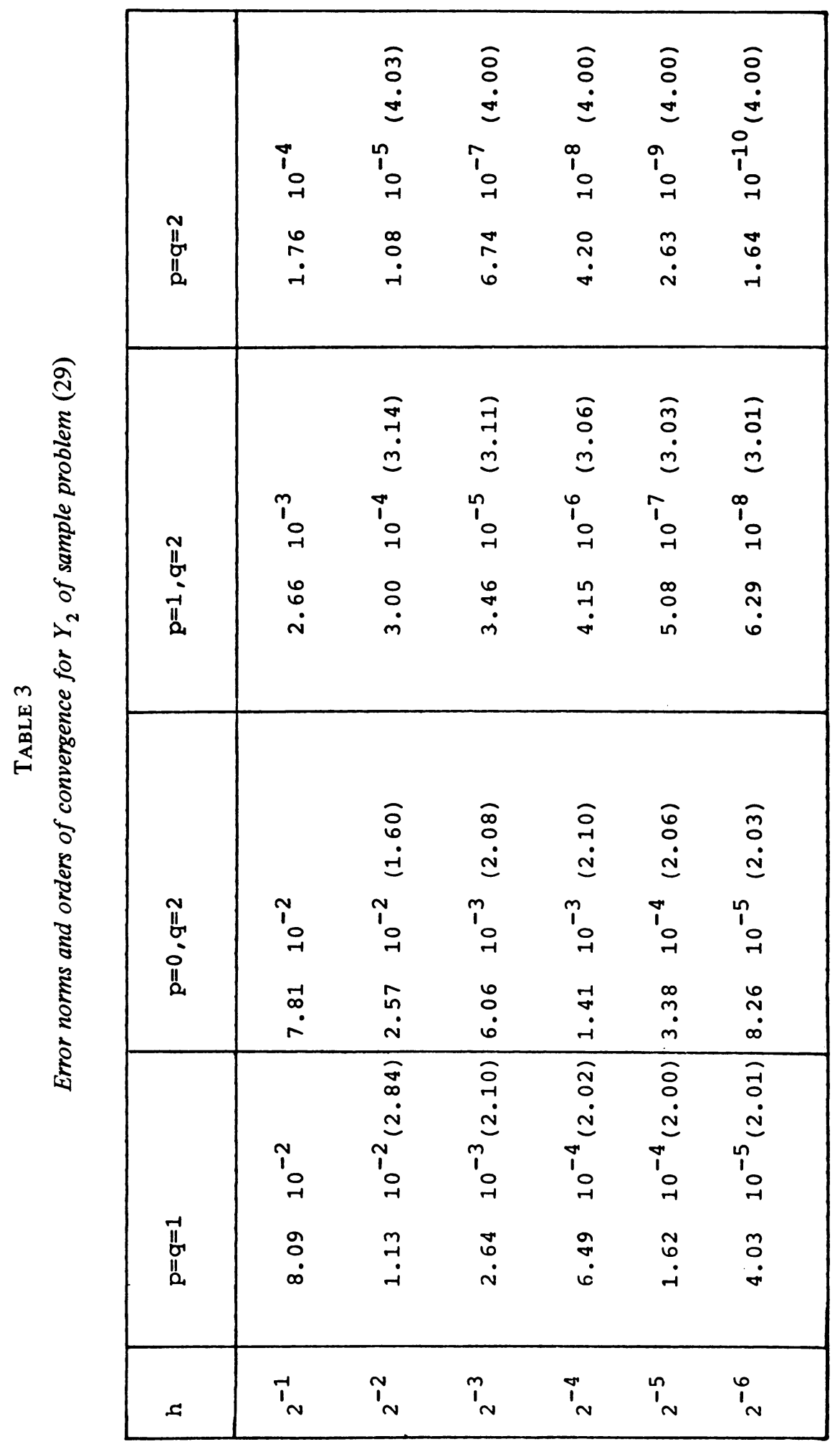


Next, we considered the system:

$$
\begin{array}{rlr}
Y_{1}^{\prime}(t)=Y_{1}^{2} Y_{2}, & Y_{1}(0)=1, & Y_{1}(t)=\exp (t), \\
Y_{2}^{\prime}(t)=-1 / Y_{1}, & Y_{2}(0)=1, & Y_{2}(t)=\exp (-t) .
\end{array}
$$

Tables 2 and 3 exhibit the discrete error norms and the computed orders of convergence for $Y_{1}$ and $Y_{2}$, respectively.

Acknowledgment. The author would like to express his indebtedness to the "Sub-Dirección de Computación" of the "Secretaria de Educación Pública" for the computing service which they have provided. He is also quite grateful to one of the referees, whose comments helped to improve the presentation of the paper.

Centro de Investigación en Matemáticas Aplicadas y en Sistemas

Universidad Nacional Autónoma de México

Apartado Postal 20-726, México 20, D. F., México

1. G. D. ANDRIA, G. D. BYRNE \& D. R. HILL, "Natural spline block implicit methods," $B I T$, v. 13, 1973. pp. 131-144. MR $48 \# 1468$.

2. G. D. ANDRIA, G. D. BYRNE \& D. R. HILL, "Integration formulas and schemes based on g-splines," Math. Comp., v. 27, 1973, pp. 831-838; addendum, ibid., microfiche supplement A1-C4. MR $49 \# 4219$.

3. J. H. ARGYRIS \& D. W. SCHARPF, "Finite elements in time and space," J. Royal Aeronautical Soc., v. 73, 1969, pp. $1041-1044$.

4. G. BIRKHOFF \& R. S. VARGA, "Discretization errors for well-set Cauchy problems. I," J. Math. and Phys., v. 44, 1965, pp. 1-23. MR 31 \#189.

5. J. C. BRUCH \& G. ZYVOLOSKI, "Finite element solution of unsteady and unsaturated flow in porous media," The Mathematics of Finite Elements and Applications, J. R. Whiteman (Editor), Academic Press, London and New York, 1973, pp. 201-211.

6. G. D. BYRNE \& D. N. H. CHI, "Linear multistep formulas based on $g$-splines," SIAM J. Numer. Anal., v. 9, 1972 , pp. 316-324. MR 46 \#10207.

7. E. D. CALLENDER, "Single step methods and low order splines for solutions of ordinary differential equations," SIAM J. Numer. Anal., v. 8, 1971, pp. 61-66. MR 47 \#4446.

8. P. J. DAVIS, Interpolation and Approximation, Blaisdell, New York, 1965.

9. G. G. DAHLQUIST, "A special stability problem for linear multistep methods," $B I T$, v. 3, 1963, pp. 27-43. MR $30 \# 715$.

10. J. DOUGLAS, JR. \& T. DUPONT, “Galerkin methods for parabolic equations", SIAM J. Numer. Anal., v. 7, 1970, pp. 575-626. MR 43 \#2863.

11. B. L. EHLE, " $A$-stable methods and Padé approximations to the exponential," $S I A M J$. Math. Anal., v. 4, 1973, pp. 671-680. MR 48 \#10119.

12. G. FIX \& N. NASSIF, "On finite element approximations to time-dependent problems," Numer. Math., v. 19, 1972, pp. 127-135. MR 46 \#10218.

13. J. P. HENNART, "Piecewise polynomials for point and space kinetics with variable reactivity," Trans. Amer. Nuclear Soc., v. 19, 1974, pp. 179-180.

14. J. P. HENNART, "Piecewise polynomial multiple collocation methods for initial value problems," Notas de Matemática y Simposia, v. 2, Sociedad Matemática Mexicana. (To appear.)

15. P. HENRICI, Discrete Variable Methods in Ordinary Differential Equations, Wiley, New York, 1962. MR 24 \#B1772.

16. B. L. HULME, "Piecewise polynomial Taylor methods for initial value problems," $\mathrm{Nu}$ mer. Math., v. 17, 1971, pp. 367-381. MR $45 \# 8002$.

17. B. L. HULME, "One-step piecewise polynomial Galerkin methods for initial value problems," Math. Comp., v. 26, 1972, pp. 415-426. MR 47 \#9834.

18. B. L. HULME, "Discrete Galerkin and related one-step methods for ordinary differential equations," Math. Comp., v. 26, 1972, pp. 881-891. MR 47 \#4448.

19. P. M. HUMMEL \& C. L. SEEBECK, JR., “A generalization of Taylor's expansion,” Amer. Math. Monthly, v. 56, 1949, pp. 243-247. MR 10, 516.

20. C. M. KANG \& K. F. HANSEN, "Finite element methods for reactor analysis," Nuclear Sci. and Engrg., v. 51, 1973, pp. 456-495. 
21. F. R. LOSCALZO \& T. D. TALBOT, "Spline function approximations for solutions of ordinary differential equations," SIAM J. Numer. Anal., v. 4, 1967, pp. 433-445. MR 36 \#4808.

22. F. R. LOSCALZO, "An introduction to the application of spline functions to initial value problems," Theory and Applications of Spline Functions (Proc. Seminar, Math. Res. Center, Univ. of Wisconsin, 1968), T.N.E. Greville (Editor), Academic Press, New York, 1969, pp. $37-64$. MR 39 \#2334.

23. J. T. ODEN, “A general theory of finite elements. Part II, Applications," Internat. J. Numer. Methods Engrg., v. 1, 1969, pp. 247-259.

24. H. S. PRICE \& R. S. VARGA, "Error bounds for semidiscrete Galerkin approximations of parabolic problems with applications to petroleum reservoir mechanics," Numerical Solutions of Field Problems in Continuum Physics (Proc. Sympos. Appl. Math., Durham, N. C., 1968), G. Birkhoff and R. S. Varga (Editors), SIAM-AMS Proc., Vol. II, Amer. Math. Soc., Providence, R. I., 1970, pp. 74-94. MR 42 \#1358.

25. B. SWARTZ \& B. WENDROFF, "Generalized finite-difference schemes," Math. Comp., v. 23, 1969, pp. 37-49. MR 39 \#1125.

26. R. S. VARGA, “On higher order stable implicit methods for solving parabolic partial differential equations," J. Math. and Phys., v. 40, 1961, pp. 220-231. MR 25 \#3613.

27. G. WARZEE, "Finite element analysis of transient heat conduction. Application of the weighted residual process," Comput. Methods Appl. Mech. Engrg., v. 3, 1974, pp. 255-268.

28. O. B. WIDLUND, "A note on unconditionally stable linear multistep methods," $B I T$, v. 7, 1967, pp. 65-70. MR 35 \#6373.

29. H. A. WRIGHT, "Some relationship between implicit Runge-Kutta, collocation and Lanczos $\tau$ methods, and their stability properties," $B I T$, v. 10, 1970, pp. 217-227.

30. O. C. ZIENKIEWICZ \& C. J. PAREKH, "Transient field problems two and three dimensional analysis by isoparametric finite elements," Internat. J. Numer. Methods Engrg., v. 2, 1970, pp. $61-71$.

31. O. C. ZIENKIEWICZ, The Finite Element Method in Engineering Science, McGraw-Hill, London and New York, 1971. MR 47 \#518. 